\title{
Bacteriological Profile and Antibiotic Susceptibility Pattern of Neonatal Septicemia and Associated Factors of ICU Hospitalization Days
}

\author{
Jinchun Li', Shiwen Xia', Yong Liu', Shasha Zhang $\mathbb{D}^{2}$, Zhengjiang Jin ${ }^{3}$ \\ 'Department of Neonatology, Maternal and Child Health Hospital of Hubei Province, Tongji Medical College, Huazhong University of Science and \\ Technology, Wuhan, 430070, China; ${ }^{2}$ Department of Pharmacy, Maternal and Child Health Hospital of Hubei Province, Tongji Medical College, \\ Huazhong University of Science and Technology, Wuhan, 430070, China; ${ }^{3}$ Department of Clinical Laboratory, Maternal and Child Health Hospital of \\ Hubei Province, Tongji Medical College, Huazhong University of Science and Technology, Wuhan, 430070, China
}

Correspondence: Shiwen Xia, Tel +86 027-65395034, Fax+86 027-87I63I33, Email shiwenxia66@I63.com; Xshiwen745@outlook.com

Purpose: To evaluate the microorganisms involved in neonatal septicemia and its antibiotic susceptibility pattern and to further investigate the factors associated with the length of intensive care unit (ICU) stay in neonatal septicemia.

Patients and Methods: A total of 297 infants with septicemia at neonatal ICU (NICU), Maternal and Child Health Hospital of Hubei Province, Tongji Medical College, Huazhong University of Science and Technology from 2016 to 2020 were enrolled. Identification of bacterial species was done using the standard positive blood culture. Data included demographics, signs at time of septicemia, laboratory values, patient sources, complications, microbiologic characteristics, and the duration of ICU stay. Univariate and multivariate gamma regression analyses were applied to determine the variables associated with ICU stay.

Results: The result demonstrated that the main causative pathogens of neonatal septicemia in our hospital were Gram-positive bacteria, among which Staphylococcus epidermidis (100 isolates, 47.17\%) was the main conditional pathogens; Escherichia coli (27 isolates, $49.09 \%$ ) was most frequently isolated among Gram-negative pathogens. Gram-positive bacteria had higher susceptibility to vancomycin, tigecycline and linezolid. Escherichia coli was susceptive to piperacillin (27/27, 100.00\%), cefotetan (27/27, 100.00\%), meropenem and imipenem (27/27, 100.00\%). Streptococcus agalactiae (95\% CI: 0.140-0.539), for patients who were transferred from other hospital (95\% CI: 0.016 to 0.354 ), septicemia during hospitalization (95\% CI: 0.411 to 0.825 ), the use of antibacterial drug during pregnancy (95\% CI: 0.362 to 2.136 ), feeding intolerance (95\% CI: 0.127 to 0.437 ), bradycardia (95\% CI: 0.196 to 0.838 ), septicemia newborns have complications (95\% CI: 0.063 to 0.291$)$, the onset age (95\% CI: 0.006 to 0.023 ), TRIPS score (95\% CI: 0.005 to 0.016$)$, and CRP level (95\% CI: 0.002 to 0.005$)$ were related to prolonged ICU stay days.

Conclusion: This study summarized common pathogens and associated drug sensitivity, and factors influencing ICU stay length. Prevention and control policy in the NICU should be strengthened.

Keywords: NICU, neonatal septicemia, microorganisms, antibiotic susceptibility

\section{Introduction}

Neonatal septicemia is defined as a clinical syndrome characterized by systemic signs and symptoms of inflammatory response following the appearance or confirmation of infection during the first month of life. ${ }^{1,2}$ It is the third most common cause of deaths among neonates, accounting for 225,000 deaths globally every year. ${ }^{3,4}$ The early symptoms and signs of neonatal septicemia infection are atypical and lack specificity, and are often similar to primary apnea, simple febrile disease, feeding intolerance, anemia and other non-infectious diseases, which are difficult to identify and easy to cause delay or overtreatment. ${ }^{5,6}$ Early screening of neonatal septicemia pathogens, early diagnosis, and reasonable use of antibiotics is very important.

Diagnosis of neonatal septicemia infection in the early hours offers better control and good patient's outcome. ${ }^{7}$ The standard method to determine whether a neonatal septicemia is in progress is a positive blood culture. ${ }^{8}$ There were 
considerable variations in septic microorganisms in the neonatal intensive care unit (NICU). In earlier studies in China, it was found that Klebsiella Pneumoniae was the most common bacteria causing neonatal septicemia, followed by Staphylococcus Aureus. ${ }^{9}$ In the United States and Australia, group B streptococcus was considered as the most common pathogenic bacteria causing neonatal septicemia. ${ }^{10,11}$ In recent years, due to frequent isolation, Acinetobacter is becoming increasingly important as a potential pathogen in neonatal septicemia. ${ }^{12}$ Some studies reported that bacterial infections associated with sepsis prolonged ICU stay. ${ }^{13,14}$ Nevertheless, there is little evidence to evaluate the clinical outcome role of microbiological characteristics in the neonatal septicemia. Because of long-term hospitalizations (mainly in ICUs), highly complex and invasive procedures, and the great need for broad-spectrum antibiotics, neonatal septicemia generates enormous expenses for the health system. ${ }^{15}$ Timely diagnosis of neonatal septicemia, reduction of antibiotic resistance, and evaluation of the influencing factors of ICU hospitalization days are of great significance for formulating appropriate treatment plans, shortening the NICU hospitalization time, and reducing the disease burden.

The aim of this study was to evaluate the microorganisms involved in neonatal septicemia and their antibiotic susceptibility pattern, and to further evaluate factors associated with the length of ICU stay in neonatal septicemia. This study may be helpful for the diagnosis of disease and the formulation of appropriate treatment plan or good supportive care for neonatal septicemia.

\section{Materials and Methods Study Design and Patients}

This study was a cohort study. A total of 297 newborns being cared for in the NICU of Maternal and Child Health Hospital of Hubei Province, Tongji Medical College, Huazhong University of Science and Technology from 2016 to 2020 were selected for this retrospective study. The inclusion criteria were as follows: (1) newborns with a clinical diagnosis of septicemia confirmed by blood culture (age $\leq 28$ days); (2) clinical data kept intact. The exclusion criteria were as follows: (1) duration of hospital stay <24 hours; (2) newborns with congenital metabolic diseases; (3) newborns with septicemia who have been treated with antimicrobials. The study follows the tenets of the Declaration of Helsinki and was approved by the Maternal and Child Health Hospital of Hubei Province, Tongji Medical College, Huazhong University of Science and Technology. The guardians of all the newborns in our department gave informed consent.

\section{Data Extraction}

The following data were extracted, including: (1) demographics: age (days), gender, birth weight $(\mathrm{g})$, body length $(\mathrm{cm})$, gestational age (weeks); (2) disease situation: onset age, parity, number of pregnancies, delivery modes, Apgar score (points), peripherally inserted central catheter (PICC), parenteral nutrition, Transport risk index of physiologic stability (TRIPS) score (points), neonatal septicemia group; (3) patient sources: obstetrics, outpatient, community, other hospital, hospital stay; (4) laboratory events: white blood cell (WBC, 109/L), red blood cell (RBC, 109/L), hemoglobin (Hb, g/L), platelet (PLT, 10/L), neutrophil (NC, 10 $/ \mathrm{L})$, lymphocytotoxicity (LYM), hematokrit (HCT, L/L), immature/total (I/T) neutrophil ratio, alanine aminotransferase (AST, U/L), total bilirubin (TBIL, $\mu \mathrm{mol} / \mathrm{L}$ ), serum albumin (ALB, g/L), seroglobulin (GLB, g/L), C-reactive protein (CRP, mg/L), procalcitonin (PCT, mg/L), NC/LYM, creatine kinase isoenzyme (CKMB, IU/L), blood urea nitrogen (BUN, mmol/L), creatinine (CR, $\mu \mathrm{mol} / \mathrm{L})$; (5) complications: jaundice, anemia, feeding intolerance, apnea, polypnea, fever, hemorrhage tendency, hepatosplenomegaly, other comorbidities; (6) outcome: duration of ICU stay.

\section{Outliers and Missing Values}

One infant had only microbiological characteristics without any other information data being deleted. In the data of the remaining 297 infants, 5 missing values in 1 minute and 5 minutes respectively in Apgar score accounted for 1.69\%, 2 missing values in standard prenatal examinations accounted for $0.68 \%$, and 4 missing values in CKMB $1.35 \%$, BUN had 3 deletions accounting for $1.01 \%$, CR had 3 deletions accounting for $1.01 \%$, I/T had 3 deletions accounting for $1.01 \%$, GLB, TBIL, ALB, ALT, AST has 1 missing value accounting for $0.34 \%$, and multiple imputation method was used to impute missing values. 


\section{Statistical Analysis}

Shapiro test was used to inspect the normality of the measurement data, and the continuous variables of the normal distribution were represented by mean \pm standard deviation (Mean \pm SD). Measurement data of non-normal distribution were exhibited as $[\mathrm{M}(\mathrm{Q} 1, \mathrm{Q} 3)]$. The enumeration data were described as the number of cases and constituent ratio $\mathrm{N}(\%)$. Gamma regression is typically used in survival analyses. When trying to explain the distribution of waiting times (or counts) with a long tail, gamma regression allows models to fit a distinct parameter to the variance of the counts that does not need to be related to the mean. We performed univariate gamma regression analysis for independent variables and multivariate gamma stepwise regression analysis for possible factors associated with the length of ICU stay. All statistical tests were conducted by two-tailed test. $P<0.05$ was considered to be statistically significant. All statistical analyses were performed using R version 4.0.3 (The R Foundation for Statistical Computing, Vienna, Austria).

\section{Results}

\section{Baseline Characteristics of Newborns with Neonatal Septicemia}

The baseline characteristics of newborns with neonatal septicemia are depicted in Table 1. A total of 297 infants were fulfilling the criteria for admission to our study, out of them, 180 (60.61\%) were male and $117(39.39 \%)$ were female. The median birth weight was $3100.00(2395.00,3400.00) \mathrm{g}$, the median length was $50.00(47.00,50.00) \mathrm{cm}$, the median gestational age was $38.57(35.57,39.86)$ weeks, and the median age was $6.00(3.00,14.00)$ days. The median TRIPS score was $1.00(0.00,13.00)$ point. Among our participants, there were 251 (84.51\%) people with a TRIPS score of $<20,152(51.18 \%)$ were early onset neonatal septicemia (EOS) and 145 (48.82\%) were late onset neonatal septicemia (LOS). In the clinical outcome, $11(3.70 \%)$ patients died, the median length of ICU stay was $10.00(8.00,16.00)$ days.

\section{Bacteriological Profile}

The primary pathogenic microorganism of neonatal septicemia was Gram-positive bacteria. Among the gram-positive bacteria, Staphylococcus epidermidis was the most common (100 isolates, 47.17\%), followed by Streptococcus agalactiae (Figure 1). Escherichia coli was found in more than 27 newborns (49.09\%) with gram-negative bacteria (Figure 2), and 18 newborns (52.94\%) were infected with Candida parapsilosis among fungus (Figure 3).

\section{Antibiotic Susceptibility Pattern}

The susceptibility rate to the following drugs was high among Gram-positive bacteria: vancomycin, tigecycline, and linezolid. None of the 27 strains of Escherichia coli were resistant to piperacillin (27/27, 100.00\%), cefotetan (27/27, $100.00 \%)$, meropenem $(27 / 27,100.00 \%)$, and imipenem $(27 / 27,100.00 \%)$. Candida parapsilosis are sensitive to all antifungal agents. The antibiotic susceptibility patterns are shown in Figure 4.

\section{Univariate Gamma Regression Analysis}

The results of the univariate analysis are depicted in Table 2 . The result showed that birth weight [ $95 \%$ confidence interval (CI): -0.086 to $0.359, P=0.234$ ], body length ( $95 \% \mathrm{CI}:-0.128$ to $-0.099, P<0.001$ ), age at onset ( $95 \% \mathrm{CI}: 0.027$ to $0.045, P<0.001$ ), Apgar score of $1 \mathrm{~min}(95 \% \mathrm{CI}:-0.438$ to $-0.211, P<0.001)$, Apgar score of $5 \mathrm{~min}(95 \% \mathrm{CI}:-0.128$ to $-0.099, P<0.001)$, PICC ( $95 \%$ CI: 1.027 to $1.441, P<0.001$ ), parenteral nutrition (95\% CI: 0.840 to $1.139, P<0.001$ ), community-derived septicemia newborn (95\% CI: -0.519 to $-0.157, P<0.001$ ), newborns with septicemia transferred from other hospital (95\% CI: 0.036 to 0.520, $P=0.027$ ), in hospital newborns ( $95 \%$ CI: 0.953 to $1.410, P<0.001$ ), septicemia newborns whose parents give multiple births ( $95 \%$ CI: 0.086 to $0.929, P=0.025)$, gestational age ( $95 \%$ CI: -0.148 to $-0.116, P<0.001)$, TRIPS score (95\% CI: 0.025 to $0.045, P<0.001$ ), severe septicemia ( $95 \%$ CI: 0.505 to $1.076, P<0.001$ ), LOS ( $95 \%$ CI: 0.481 to $0.848, P<0.001$ ) were associated with length of stay in the ICU.

Laboratory indicators such as the levels of RBC (95\% CI: -0.513 to $-0.232, P<0.001)$, $\mathrm{Hb}(95 \% \mathrm{CI}:-0.015$ to $-0.008, P<0.001)$, PLT (95\% CI: -0.003 to $-0.001, P<0.001)$, LYM (95\% CI: $-0.125,-0.028, P=0.006)$, HCT (95\% CI: $-4.775,-2.283, P<0.001)$, I/T (95\% CI: $0.785,2.035, P<0.001)$, TBIL (95\% CI: -0.005 to $-0.003, P<0.001)$, ALB (95\% 
Table I Baseline Characteristics of Included Newborns

\begin{tabular}{|c|c|}
\hline Variables & Total $(n=297)$ \\
\hline \multicolumn{2}{|l|}{ Gender, n (\%) } \\
\hline Male & $180(60.61 \%)$ \\
\hline Female & 117 (39.39\%) \\
\hline Birth weight, $M\left(Q_{1}, Q_{3}\right),(g)$ & $3100.00(2395.00,3400.00)$ \\
\hline Body length, $M\left(Q_{1}, Q_{3}\right),(\mathrm{cm})$ & $50.00(47.00,50.00)$ \\
\hline Gestational age, $M\left(Q_{1}, Q_{3}\right),($ Week $)$ & $38.57(35.57,39.86)$ \\
\hline Onset age, $M\left(Q_{1}, Q_{3}\right)$, (Day) & $6.00(3.00,14.00)$ \\
\hline Apgar Score I min $M\left(Q_{1}, Q_{3}\right)$, (Points) & $9.00(8.00,10.00)$ \\
\hline Apgar Score $5 \mathrm{~min}, \mathrm{M}\left(\mathrm{Q}_{1}, \mathrm{Q}_{3}\right)$, (Points) & $10.00(9.00,10.00)$ \\
\hline PICC (Yes) & $50(16.84 \%)$ \\
\hline Parenteral nutrition, (Yes) & I33 (44.78\%) \\
\hline \multicolumn{2}{|l|}{ Patient sources } \\
\hline Obstetrics & 108 (36.36\%) \\
\hline Outpatient & $2(0.67 \%)$ \\
\hline Community & $100(33.67 \%)$ \\
\hline Other hospital & $40(13.47 \%)$ \\
\hline Hospital stay & 47 (15.82\%) \\
\hline TRIPS Score, $M\left(Q_{1}, Q_{3}\right)$, (Points) & $1.00(0.00,13.00)$ \\
\hline \multicolumn{2}{|l|}{ TRIPS Groups, n (\%) } \\
\hline Severe (TRIPS Score $\geq 20$ ) & $46(15.49 \%)$ \\
\hline Non-severe (TRIPS Score < 20) & $251(84.51 \%)$ \\
\hline \multicolumn{2}{|l|}{ Neonatal sepsis group, $\mathrm{n}(\%)$} \\
\hline EOS & $152(51.18 \%)$ \\
\hline LOS & 145 (48.82\%) \\
\hline Jaundice, n (\%), (Yes) & $153(51.52 \%)$ \\
\hline Anemia, n (\%), (Yes) & $84(28.28 \%)$ \\
\hline Feeding intolerance, $n$ (\%), (Yes) & $40(13.47 \%)$ \\
\hline Apnea, n (\%), (Yes) & 38 (12.79\%) \\
\hline Polypnea, n (\%), (Yes) & $88(29.63 \%)$ \\
\hline Fever, n (\%), (Yes) & 105 (35.35\%) \\
\hline Hemorrhage tendency, n (\%), (Yes) & $28(9.43 \%)$ \\
\hline Hepatosplenomegaly, n (\%), (Yes) & 15 (5.05\%) \\
\hline Other comorbidities, n (\%), (Yes) & $193(64.98 \%)$ \\
\hline \multicolumn{2}{|l|}{ Outcomes } \\
\hline \multicolumn{2}{|l|}{ Survival state, $n$ (\%) } \\
\hline Survival & $286(96.30 \%)$ \\
\hline Death & II (3.70\%) \\
\hline Duration of ICU stay, $M\left(Q_{1}, Q_{3}\right)$, (day) & $10.00(8.00,16.00)$ \\
\hline Hospitalization costs, $M\left(Q_{1}, Q_{3}\right)$, (yuan) & $16187.88(11348.24,37449.08)$ \\
\hline
\end{tabular}

Abbreviations: PICC, peripherally inserted central catheter; EOS, early-onset neonatal septicemia; LOS, late-onset neonatal septicemia; ICU, intensive care unit.

CI: -0.101 to $-0.067, P<0.001)$, GLB (95\% CI: -0.062 to $-0.018, P=0.002)$, CRP (95\% CI: 0.001 to $0.010, P=0.005$ ), BUN (95\% CI: 0.034 to $0.124, P<0.001)$ were found to be related to duration of ICU stay.

The result showed that duration of ICU was associated with microbiological characteristics, including Staphylococcus epidermidis (95\% CI: -0.911 to $-0.524, P<0.001)$, Escherichia coli (95\% CI: -0.776 to $-0.031, P=0.026)$, Candida parapsilosis (95\% CI: 0.635 to $1.509, P<0.001$ ), Staphylococcus hominis (95\% CI: -0.866 to $-0.001, P=0.037$ ).

Among the symptoms, jaundice $(95 \% \mathrm{CI}:-0.739,-0.327, P<0.001)$, abnormal body temperature (95\% CI: 0.201 , $0.654, P<0.001$ ), anemia (95\% CI: 0.579 to $1.006, P<0.001$ ), feeding intolerance (95\% CI: 0.344 to $0.972, P<0.001)$, apnea (95\% CI: 0.713 to $1.287, P<0.001)$, polypnea (95\% CI: 0.228 to $0.698, P<0.001$ ), fever (95\% CI: 0.146 to 0.605 , $P=0.002$ ), bleeding tendency (95\% CI: 0.106 to $0.846, P=0.016$ ), tachycardia (95\% CI: 0.244 to $0.860, P<0.001$ ), decreased responsiveness ( $95 \% \mathrm{CI}: 0.338$ to $0.824, P<0.001)$, cyanosis ( $95 \% \mathrm{CI}: 0.470$ to $0.898, P<0.001)$, rash $(95 \% \mathrm{CI}$ : -0.325 to $0.557, P<0.001$ ) have been found to influence the length of stay in the ICU. 

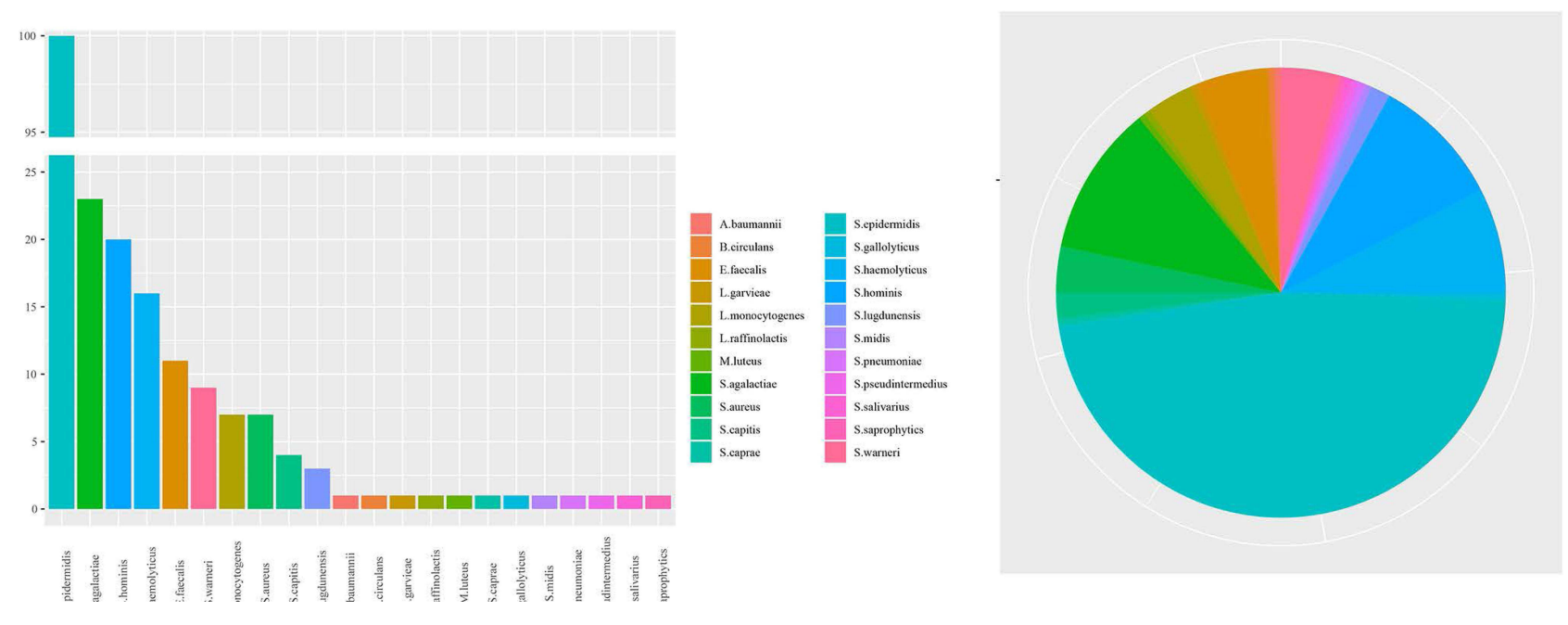

Figure I Frequency of neonatal septicemia with Gram-positive bacteria.
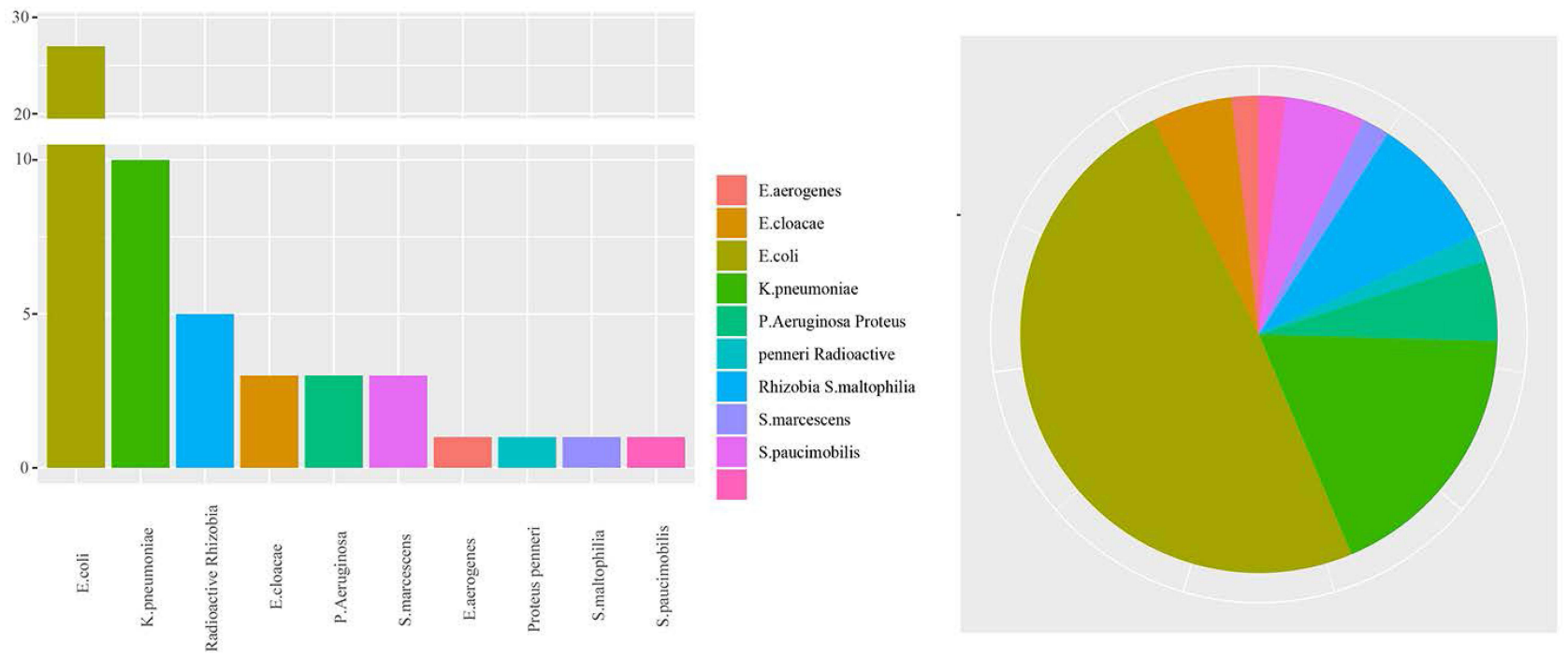

Figure 2 Frequency of neonatal septicemia with Gram-negative bacteria.

\section{Multivariate Gamma Regression}

Results of multivariate gamma regression are shown in Table 3. The result showed that the body length (95\% CI: -0.044 to -0.004$)$, low blood pressure ( $95 \% \mathrm{CI}$ : -0.590 to -0.102$)$, gestational age ( $95 \% \mathrm{CI}$ : -0.059 to -0.009 ), the lever of PCT (95\% CI: -0.005 to -0.001$)$, sources of patients in community patients (95\% CI: -0.333 to -0.039$)$, twins $(95 \%$ CI: -2.139 to -0.365$)$, CKMB (95\% CI: -0.001 to 0.000 ) were associated with the number of days in ICU. The number of hospital stays decreased with each unit increase in the value of these variables.

For patients who were transferred from other hospital (95\% CI: 0.016 to 0.354), septicemia during hospitalization ( $95 \%$ CI: 0.411 to 0.825 ), the use of antibacterial drug during pregnancy (95\% CI: 0.362 to 2.136 ), feeding intolerance (95\% CI: 0.127 to 0.437 ), bradycardia (95\% CI: 0.196 to 0.838 ), septicemia newborns have complications (95\% CI: 0.063 to 0.291 ), the onset age ( $95 \%$ CI: 0.006 to 0.023 ), TRIPS score (95\% CI: 0.005 to 0.016$)$, Streptococcus agalactiae (95\% CI: $0.14-0.539)$ and CRP level (95\% CI: 0.002 to 0.005) were also related to ICU stay days. The number of hospital stays increased when these variables increased by one unit. 

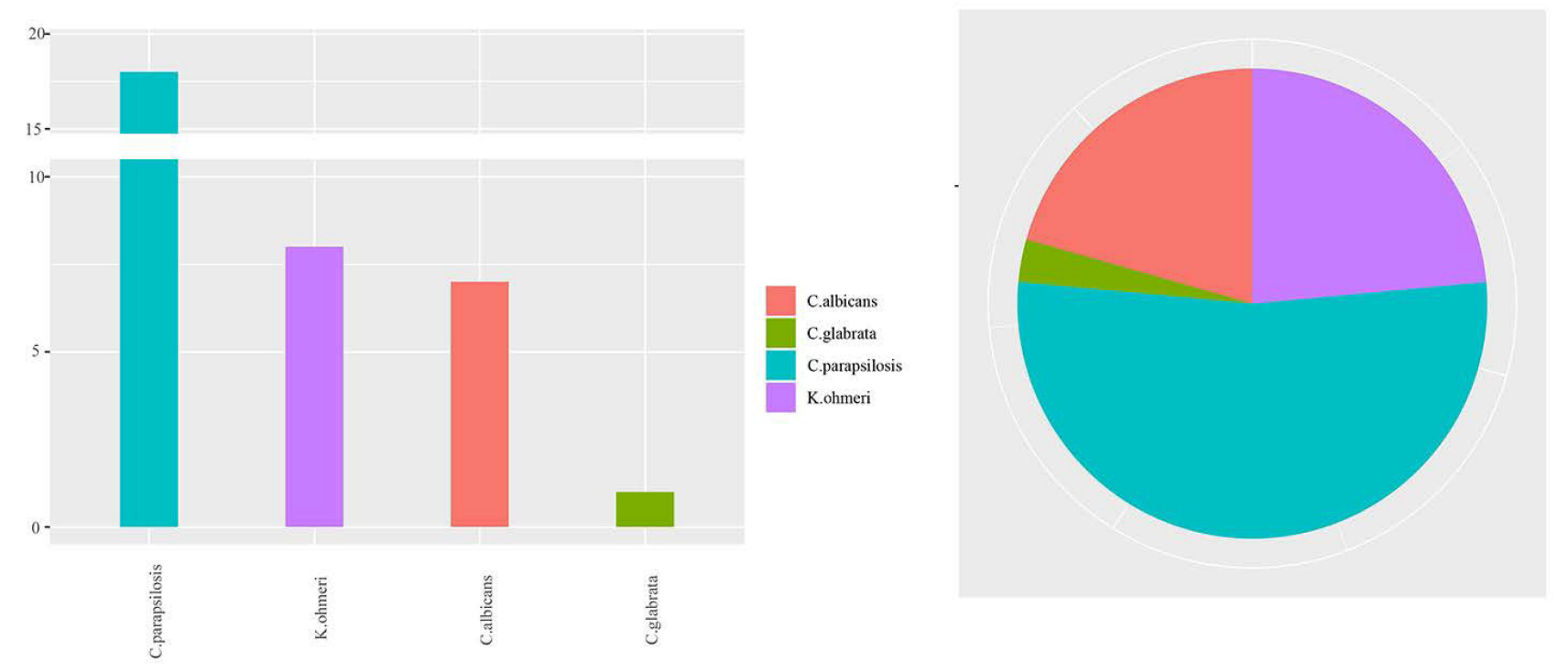

Figure 3 Frequency of neonatal septicemia with fungus.

A

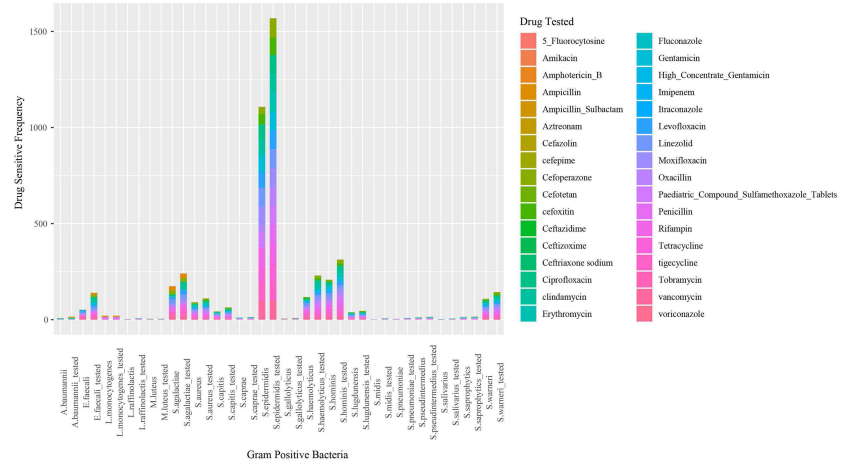

B

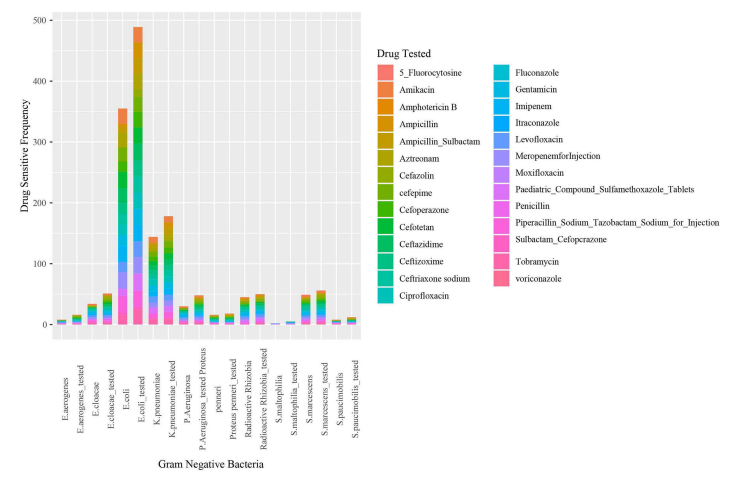

C



Figure 4 Drug sensitivity for Gram-positive bacteria, Gram-negative bacteria and fungus; (A) drug sensitivity for Gram-positive bacteria; (B) drug sensitivity for Gramnegative bacteria; (C) drug sensitivity for fungus.

\section{Discussion}

Neonatal septicemia is one of the public health issues of global concern, and one of the main problems and challenges faced by NICU. ${ }^{7}$ It is also an important reason for the high neonatal mortality rate and prolonged hospital stay. ${ }^{16}$ Timely and appropriate diagnoses are particularly important for lowering morbidity, mortality, and hospitalization time. ${ }^{17}$ The 
Table 2 Univariate Gamma Regression Results

\begin{tabular}{|c|c|c|c|}
\hline Variables & $\beta$ & $95 \% \mathrm{Cl}$ & $\mathbf{P}$ \\
\hline \multicolumn{4}{|l|}{ Baseline information } \\
\hline Gender (female) & 0.135 & $(-0.086,0.359)$ & 0.234 \\
\hline Birth weight (g) & -0.001 & $(-0.001,-0.001)$ & $<0.001$ \\
\hline Body length $(\mathrm{cm})$ & -0.113 & $(-0.128,-0.099)$ & $<0.001$ \\
\hline Onset age & 0.036 & $(0.027,0.045)$ & $<0.001$ \\
\hline Apgar Score I min (points) & -0.321 & $(-0.438,-0.211)$ & $<0.001$ \\
\hline Apgar Score 5 min (points) & -0.113 & $(-0.128,-0.099)$ & $<0.001$ \\
\hline PICC (Yes) & 1.229 & $(I .027,1.44 I)$ & $<0.001$ \\
\hline Parenteral nutrition (Yes) & 0.989 & $(0.840,1.139)$ & $<0.001$ \\
\hline \multicolumn{4}{|l|}{ Patient sources } \\
\hline Obstetrics & REF & & \\
\hline Outpatient & -0.416 & $(-1.227,0.679)$ & 0.382 \\
\hline Community & -0.338 & $(-0.519,-0.157)$ & $<0.001$ \\
\hline Other hospital & 0.274 & $(0.036,0.520)$ & 0.027 \\
\hline Hospital stay & 1.178 & $(0.953,1.410)$ & $<0.001$ \\
\hline Standardized prenatal care & -0.139 & $(-0.497,0.189)$ & 0.425 \\
\hline \multicolumn{4}{|l|}{ Number of pregnancies } \\
\hline Multiple & REF & & \\
\hline Single & -0.16 & $(-0.380,0.062)$ & 0.157 \\
\hline \multicolumn{4}{|l|}{ Parity } \\
\hline Single & REF & & \\
\hline Multiple & 0.482 & $(0.086,0.929)$ & 0.025 \\
\hline Twins & -0.923 & $(-2.37 \mathrm{I}, \mathrm{I} .805)$ & $0.34 I$ \\
\hline \multicolumn{4}{|l|}{ Delivery modes } \\
\hline Cesarean & REF & & \\
\hline Vaginal delivery & -0.125 & $(-0.353,0.098)$ & 0.277 \\
\hline Preeclampsia (Yes) & 0.469 & $(-0.35 \mathrm{I}, \mathrm{I} .585)$ & 0.331 \\
\hline Premature rupture of membrane (Yes) & 0.146 & $(-0.228,0.564)$ & 0.469 \\
\hline Fever (Yes) & 0.136 & $(-0.239,0.555)$ & 0.5 \\
\hline Antibacterial drug use during pregnancy (Yes) & 0.743 & $(-0.706,3.473)$ & 0.444 \\
\hline Antenatal hemorrhage (Yes) & 0.956 & $(0.031,2.277)$ & 0.086 \\
\hline Gestational age (Week) & -0.132 & $(-0.148,-0.116)$ & $<0.001$ \\
\hline Complications (Yes) & 0.835 & $(0.649,1.018)$ & $<0.001$ \\
\hline \multicolumn{4}{|l|}{ Disease severity } \\
\hline TRIPS score (points) & 0.035 & $(0.025,0.045)$ & $<0.001$ \\
\hline \multicolumn{4}{|l|}{ TRIPS groups } \\
\hline Severe (Trips score $\geq 20$ ) & 0.781 & $(0.505,1.076)$ & $<0.001$ \\
\hline \multicolumn{4}{|l|}{ Neonatal sepsis groups } \\
\hline EOS & REF & & \\
\hline LOS & 0.665 & $(0.48 \mathrm{I}, 0.848)$ & $<0.001$ \\
\hline \multicolumn{4}{|l|}{ Laboratory value } \\
\hline WBC $\left(10^{9} / \mathrm{L}\right)$ & -0.006 & $(-0.018,0.006)$ & 0.454 \\
\hline $\operatorname{RBC}\left(10^{9} / \mathrm{L}\right)$ & -0.372 & $(-0.5 \mid 3,-0.232)$ & $<0.001$ \\
\hline $\mathrm{Hb}(\mathrm{g} / \mathrm{L})$ & -0.011 & $(-0.015,-0.008)$ & $<0.001$ \\
\hline $\operatorname{PLT}\left(10^{9} / \mathrm{L}\right)$ & -0.002 & $(-0.003,-0.001)$ & $<0.001$ \\
\hline $\mathrm{NC}\left(10^{9} / \mathrm{L}\right)$ & -0.001 & $(-0.016,0.016)$ & 0.943 \\
\hline $\operatorname{LYM}\left(10^{9} / \mathrm{L}\right)$ & -0.077 & $(-0.125,-0.028)$ & 0.006 \\
\hline HCT (L/L) & -3.53 & $(-4.775,-2.283)$ & $<0.001$ \\
\hline $\mathrm{I} / \mathrm{T}$ neutrophil ratio & 1.402 & $(0.785,2.035)$ & $<0.001$ \\
\hline ALT (U/L) & 0.001 & $(-0.002,0.005)$ & 0.597 \\
\hline AST (U/L) & 0 & $(-0.001,0.001)$ & 0.77 \\
\hline
\end{tabular}


Table 2 (Continued).

\begin{tabular}{|c|c|c|c|}
\hline Variables & $\beta$ & $95 \% \mathrm{Cl}$ & $\boldsymbol{P}$ \\
\hline TBIL $(\mu \mathrm{mol} / \mathrm{L})$ & -0.004 & $(-0.005,-0.003)$ & $<0.001$ \\
\hline ALB $(g / L)$ & -0.084 & $(-0.101,-0.067)$ & $<0.001$ \\
\hline GLB $(g / L)$ & -0.04 & $(-0.062,-0.018)$ & 0.002 \\
\hline $\mathrm{CRP}(\mathrm{mg} / \mathrm{L})$ & 0.005 & $(0.001,0.010)$ & 0.005 \\
\hline PCT (mg/L) & -0.002 & $(-0.006,0.004)$ & 0.475 \\
\hline NC/LYM & 0.001 & $(-0.021,0.029)$ & 0.964 \\
\hline CKMB (IU/L) & 0 & $(-0.001,0.001)$ & 0.568 \\
\hline BUN (mmol/L) & 0.077 & $(0.034,0.124)$ & $<0.001$ \\
\hline $\mathrm{Cr}(\mu \mathrm{mol} / \mathrm{L})$ & 0.003 & $(-0.003,0.009)$ & 0.33 \\
\hline \multicolumn{4}{|l|}{ Microbiology features } \\
\hline Staphylococcus epidermidis (Yes) & -0.719 & $(-0.911,-0.524)$ & $<0.001$ \\
\hline Escherichia coli (Yes) & -0.422 & $(-0.776,-0.03 \mathrm{I})$ & 0.026 \\
\hline Candida parapsilosis (Yes) & 1.044 & $(0.635,1.509)$ & $<0.001$ \\
\hline Staphylococcus haemolyticus (Yes) & -0.001 & $(-0.454,0.524)$ & 0.998 \\
\hline Staphylococcus hominis (Yes) & -0.46 & $(-0.866,-0.00 \mathrm{I})$ & 0.037 \\
\hline Streptococcus agalactiae (Yes) & -0.044 & $(-0.432,0.391)$ & 0.833 \\
\hline \multicolumn{4}{|l|}{ Symptom } \\
\hline Jaundice (Yes) & -0.533 & $(-0.739,-0.327)$ & $<0.001$ \\
\hline Anemia (Yes) & 0.79 & $(0.579,1.006)$ & $<0.001$ \\
\hline Feeding intolerance (Yes) & 0.646 & $(0.344,0.972)$ & $<0.001$ \\
\hline Apnea (Yes) & 0.99 & $(0.713,1.287)$ & $<0.001$ \\
\hline Polypnea (Yes) & 0.459 & $(0.228,0.698)$ & $<0.001$ \\
\hline Fever (Yes) & 0.373 & $(0.146,0.605)$ & 0.002 \\
\hline Hemorrhage tendency (Yes) & 0.457 & $(0.106,0.846)$ & 0.016 \\
\hline Hepatosplenomegaly (Yes) & 0.305 & $(-0.162,0.847)$ & 0.234 \\
\hline Tachycardia (Yes) & $0.54 I$ & $(0.244,0.860)$ & $<0.001$ \\
\hline Bradycardia (Yes) & -0.219 & $(-0.973,0.39)$ & 0.523 \\
\hline Hypotension (Yes) & 0.033 & $(-0.433,0.576)$ & 0.896 \\
\hline Decreased responsiveness (Yes) & 0.576 & $(0.338,0.824)$ & $<0.001$ \\
\hline Cyanosis (Yes) & 0.682 & $(0.470,0.898)$ & $<0.001$ \\
\hline Emesis (Yes) & 0.03 & $(-0.339,0.440)$ & $0.88 I$ \\
\hline Rash (Yes) & -0.451 & $(-0.706,-0.182)$ & $<0.001$ \\
\hline Tic (Yes) & 0.088 & $(-0.325,0.557)$ & 0.696 \\
\hline
\end{tabular}

Abbreviations: PICC, peripherally inserted central catheter; EOS, early-onset neonatal septicemia; LOS, late-onset neonatal septicemia; WBC, white blood cell; RBC, red blood cell; Hb, hemoglobin; PLT, platelet; NC, neutrophil; LYM, lymphocytotoxicity; HCT, hematokrit; I/T, immature/total; ALT, alanine aminotransferase; AST, aspartate aminotransferase; TBIL, total bilirubin; ALB, serum albumin; GLB, seroglobulin; CRP, C-reactive protein; PCT, procalcitonin; $\mathrm{CKMB}$, creatine kinase isoenzyme; $\mathrm{BUN}$, blood urea nitrogen; $\mathrm{Cr}$, creatinine.

present study was aimed to determine the pathogen and antimicrobial susceptibility of neonatal septicemia, and to explore the influencing factors of ICU hospitalization days. The result demonstrated that the main causative pathogens of neonatal septicemia in our hospital were Gram-positive bacteria, among which Staphylococcus epidermidis was the main conditional pathogens; and among Gram-negative pathogens, Escherichia coli was most frequently isolated. Grampositive bacteria had higher susceptibility to vancomycin, tigecycline and linezolid. Escherichia coli were susceptive to piperacillin, cefotetan, meropenem, and imipenem. Streptococcus agalactiae, for patients who were transferred from other hospital, septicemia during hospitalization, the use of antibacterial drug during pregnancy, feeding intolerance, bradycardia, septicemia newborns have complications, the onset age, TRIPS score, and CRP level were associated with increased risk of ICU stay.

The result of our study showed that Gram-positive infection was found to be more common than Gram-negative and fungal infection, which was supported by multiple studies. ${ }^{5,18,19}$ Sharma et al found that Gram-positive bacteria were the most common cause of septicemia. ${ }^{18}$ In our study, Gram-positive bacteria have a higher sensitivity to vancomycin, 
Table 3 Multivariate Gamma Regression Results

\begin{tabular}{|c|c|c|c|}
\hline Variables & $\boldsymbol{\beta}$ & $95 \% \mathrm{Cl}$ & $\boldsymbol{P}$ \\
\hline \multicolumn{4}{|l|}{ Baseline information } \\
\hline Body length & -0.024 & $(-0.044,0.004)$ & 0.02 \\
\hline \multicolumn{4}{|l|}{ Patient source } \\
\hline Obstetric & REF & & \\
\hline Outpatient & -0.107 & $(0.618,0.496)$ & 0.707 \\
\hline Community & -0.187 & $(-0.333,-0.039)$ & 0.016 \\
\hline Other hospital & 0.183 & $(0.016,0.354)$ & 0.035 \\
\hline Hospital stay & 0.617 & $(0.4 \mathrm{II}, 0.825)$ & $<0.001$ \\
\hline \multicolumn{4}{|l|}{ Parity } \\
\hline Single & REF & & \\
\hline Multiple & -0.08 & $(-0.264,0.111)$ & 0.396 \\
\hline Twins & -1.325 & $(-2.139,0.365)$ & 0.003 \\
\hline Antibacterial drug use during pregnancy (Yes) & 1.177 & $(0.362,2.136)$ & 0.009 \\
\hline Feeding intolerance(Yes) & 0.28 & $(0.127,0.437)$ & $<0.001$ \\
\hline Bradycardia (Yes) & 0.529 & $(0.196,0.838)$ & 0.002 \\
\hline Hypotension (Yes) & -0.35 & $(-0.590,-0.102)$ & 0.01 \\
\hline Comorbidity (Yes) & 0.177 & $(0.063,0.291)$ & 0.003 \\
\hline Gestational week & -0.034 & $(-0.059,-0.009)$ & 0.006 \\
\hline Onset age & 0.014 & $(0.006,0.023)$ & 0.001 \\
\hline \multicolumn{4}{|l|}{ Disease severity } \\
\hline TRIPS score & 0.01 & $(0.005,0.016)$ & $<0.001$ \\
\hline \multicolumn{4}{|l|}{ Neonatal sepsis groups } \\
\hline EOS & REF & & \\
\hline LOS & -0.021 & $(-0.184,0.142)$ & 0.804 \\
\hline \multicolumn{4}{|l|}{ Microbiology features } \\
\hline Staphylococcus epidermidis (Yes) & 0.011 & $(-0.118,0.140)$ & 0.861 \\
\hline Escherichia coli (Yes) & -0.086 & $(-0.266,0.098)$ & 0.356 \\
\hline Candida parapsilosis (Yes) & 0.035 & $(-0.195,0.27)$ & 0.765 \\
\hline Staphylococcus haemolyticus (Yes) & 0.098 & $(-0.109,0.315)$ & 0.363 \\
\hline Staphylococcus hominis (Yes) & -0.133 & $(-0.335,0.076)$ & 0.205 \\
\hline Streptococcus agalactiae (Yes) & 0.337 & $(0.140,0.539)$ & 0.001 \\
\hline \multicolumn{4}{|l|}{ Laboratory value } \\
\hline CRP & 0.003 & $(0.002,0.005)$ & $<0.001$ \\
\hline PCT & -0.003 & $(-0.005,-0.001)$ & 0.014 \\
\hline CKMB & -0.001 & $(-0.001,0.00)$ & $<0.001$ \\
\hline
\end{tabular}

Abbreviations: EOS, early-onset neonatal septicemia; LOS, late-onset neonatal septicemia; CRP, C-reactive protein; PCT, procalcitonin; CKMB, creatine kinase isoenzyme.

tigecycline, and linezolid. A study ${ }^{20}$ evaluating the changing trend of neonatal septicemia and antibiotic susceptibility pattern of isolates in Nepal has found that vancomycin showed $100 \%$ sensitivity towards gram-positive isolates similar to the findings of Muley et al. ${ }^{21} \mathrm{Li}$ et al found Escherichia coli was the most common Gram-negative bacteria in neonatal septicemia, ${ }^{22}$ which was consistent with our findings. Our results demonstrated that Escherichia coli was $100 \%$ sensitive to piperacillin, cefotetan, meropenem, and imipenem. These drugs might be able to apply to neonatal sepsis patients infected with Escherichia coli. Ballot et $\mathrm{al}^{23}$ found that all the Escherichia coli's tested susceptible to the 3rd-generation cephalosporins. The relationship between antimicrobial use and drug resistance is complex. Possible factors involved in the observed pattern of antibiotic resistance include pathogen-drug interactions, pathogen-host interactions, pathogen mutation rates and transmission rate of pathogens to babies from mother, other babies or the environment. ${ }^{24}$ The ideal choice for antimicrobial drugs is to cover the most common pathogens without providing selection pressure for antibiotic 
resistance. ${ }^{25}$ Ongoing surveillance of microbiological isolates and their sensitivity patterns is therefore an essential part of antibiotic stewardship.

Of the assessed variables, the length of hospital stay showed significant association with the presence of Streptococcus agalactiae in the present study, which is supported by a study ${ }^{26}$ that found that the clinical symptoms of neonatal Streptococcus agalactiae sepsis are atypical, with significant changes in inflammatory indicators, long hospital stay and high costs. Streptococcus agalactiae colonizes the gastrointestinal and genital tracts of around $20 \%$ of pregnant women. ${ }^{27}$ Transmission from colonized mothers to their infants can occur before or during birth, and can result in early onset disease (in the first 6 days of life), which may present with pneumonia, respiratory failure, or even rapidly progressing bacteremia and septic shock. It has high morbidity rate, fatality rate and disability rate; late-onset disease (7-89 days of age) can result from vertical transmission but also from nosocomial or community transmission, which is usually characterized by high fever as the first symptom, is easy to be combined with purulent meningitis, which can lead to serious neurological sequelae and irreversible damage such as hearing loss. ${ }^{4,28}$ Streptococcus agalactiae was reported to be likely to cause severe sepsis in neonates. ${ }^{29}$ Intravenous antibiotics given to the mother during labor can prevent early-onset disease, and intrapartum antibiotic prophylaxis has been adopted in many countries. ${ }^{30,31}$ Such strategies are based either on identifying at-risk women using swab-based screening or on the presence of clinical risk factors. ${ }^{32}$ However, intrapartum antibiotic prophylaxis does not prevent late onset disease. ${ }^{33}$ Globally, surveillance data demonstrated a uniform susceptibility of Streptococcus agalactiae strains to penicillin/ampicillin and most $\beta$-lactams for more than 50 years. ${ }^{34}$ However, the emergence of strains with reduced susceptibility to penicillin has been very recently reported. ${ }^{35}$ The increasing number of multi-resistant strains is a serious matter of concern. Newborns are in transition from a sterile intrauterine environment to a coexistence with microorganisms, which probably facilitates expansion and invasion of potentially pathogenic bacteria. ${ }^{36}$ The neonatal immune system is vulnerable due to characteristics including decreased cellular activity, underdeveloped complement systems, preferential anti-inflammatory responses, and insufficient pathogenic memory. ${ }^{37}$ Several components of the clearance apparatus, such as directed movement of the microorganism, expression of adhesion molecules, phagocytosis and the intracellular killing of Streptococcus agalactiae, are also deficient in newborns. ${ }^{38,39}$ Excessive inflammatory response, clinical manifestations, severity of complications of streptococcus agalactiae septicemia, antibiotic resistance, and neonatal self-limited conditions possibly contribute to prolonged hospital stay in neonatal Streptococcus agalactiae sepsis. In addition, neonatal Streptococcus agalactiae sepsis can lead to a low level of WBC in newborns, and WBC level is correlated with the severity and prognosis of the disease. ${ }^{40}$ In clinical practice, attention should be paid to neonatal Streptococcus agalactiae sepsis, and various methods should be adopted to improve the detection rate of neonatal Streptococcus agalactiae sepsis, antibiotic treatment should be given in time, so as to reduce the length of stay in ICU and related complications or adverse outcomes.

Although the transfer to a high-level NICU is necessary for critically ill newborns to receive specialized care, a transfer is potentially hazardous and is associated with a very high level of risk. ${ }^{41}$ Transfers may be associated with adverse outcomes, including delays in care, increased likelihood of ICU stay, and longer lengths of stay. ${ }^{42}$ In this study, patients who were transferred from another hospital had a longer ICU stay. Similarly, a study evaluating transfer hospitalizations for pediatric severe sepsis or septic shock has found that transferred children had longer hospitalization and accrued higher charges than non-transferred children. ${ }^{43} \mathrm{~A}$ study showed that remoteness of the referral hospital and longer duration of transport are associated with increased hospital lengths of stay. ${ }^{44}$ Besides, longer hospitalization among the transfer hospitalizations appeared commensurate with the severity of patient illness. ${ }^{43}$ We also found that TRIPS score, which was used to assess the clinical severity of infants at the time transport ${ }^{45}$ was performed, was related to an increased hospital length of stay. Knowledge of the transfer process and the severity of disease risk associated with the transfer provides information on the assessment of the neonatal transport and may reduce the length of stay and decrease adverse outcomes.

A key strength of this study is the data collection using a consistent gold standard for the diagnosis of neonatal septicemia, which makes our results more credible. This study has to be seen in light of some limitations; first, this was a single-center study with a relatively small sample size, which might either lack the scientific precision or external validity needed to assist widespread changes in practice. Second, we did not monitor long-term outcomes in patients with neonatal sepsis. Herein, long-term, multicenter, and prospective clinical studies are therefore suggested in future. 


\section{Conclusion}

Our study demonstrated that Staphylococcus epidermidis, Escherichia coli, and Candida parapsilosis remain to be the principal organisms responsible for neonatal septicemia in the ICU. Gram-positive bacteria and Escherichia coli had a higher proportion of drug sensitivity. Besides, Streptococcus agalactiae was associated with increased risk of ICU stay. This study summarized common pathogens and associated drug sensitivity, and factors influencing ICU stay length, prompting us to strengthen nosocomial infection control and formulate discriminating plans to prevent infections, reducing morbidity and length of ICU stay.

\section{Disclosure}

The authors report no conflicts of interest in this work.

\section{References}

1. Kim F, Polin RA, Hooven TA. Neonatal sepsis. BMJ. 2020;371:m3672. doi:10.1136/bmj.m3672

2. Shane AL, Sánchez PJ, Stoll BJ. Neonatal sepsis. Lancet. 2017;390:1770-1780. doi:10.1016/S0140-6736(17)31002-4

3. Chaurasia S, Sivanandan S, Agarwal R, et al. Neonatal sepsis in South Asia: huge burden and spiralling antimicrobial resistance. BMJ. 2019;364: k5314. doi:10.1136/bmj.k5314

4. Narava S, Rajaram G, Ramadevi A, et al. Prevention of perinatal group B streptococcal infections: a review with an Indian perspective. Indian J Med Microbiol. 2014;32:6-12. doi:10.4103/0255-0857.124286

5. Dong H, Cao H, Zheng H. Pathogenic bacteria distributions and drug resistance analysis in 96 cases of neonatal sepsis. BMC Pediatr. 2017;17:44. doi:10.1186/s12887-017-0789-9

6. Pokhrel B, Koirala T, Shah G, et al. Bacteriological profile and antibiotic susceptibility of neonatal sepsis in neonatal intensive care unit of a tertiary hospital in Nepal. BMC Pediatr. 2018;18:208. doi:10.1186/s12887-018-1176-x

7. Shatalov A, Mangue P, Foqahaa RJ. Predominance of multi-drug resistant Klebsiella pneumonia and other gram negative bacteria in neonatal sepsis in Equatorial Guinea. Open J Med Microbiol. 2015;5:254-258. doi:10.4236/ojmm.2015.54031

8. Weldu Y, Naizgi M, Hadgu A, et al. Neonatal septicemia at intensive care unit, Ayder Comprehensive Specialized Hospital, Tigray, North Ethiopia: bacteriological profile, drug susceptibility pattern, and associated factors. PLoS One. 2020;15:e0235391. doi:10.1371/journal.pone.0235391

9. NNPD Network. National Neonatal-Perinatal Database-Report 2002-2003. New Delhi, India: National Neonatology Forum NNPD Network. 2005.

10. Puopolo KM. Bacterial and fungal infections. Cloherty JP, Eichenwald EC, Stark AR, editors. In: Manual of Neonatal Care. 6th ed. Philadelphia, PA: Lippincott William and Wilkins; 2008:274-300.

11. Bartlett AW, Smith B, George CR, et al. Epidemiology of late and very late onset group B Streptococcal disease: fifteen-year experience from two Australian tertiary pediatric facilities. Pediatr Infect Dis J. 2017;36:20-24. doi:10.1097/INF.0000000000001345

12. De AS, Rathi MR, Mathur MM. Mortality audit of neonatal sepsis secondary to Acinetobacter. J Glob Infect Dis. 2013;5:3-7. doi:10.4103/0974777X.107165

13. Anitha A, Babu K, Siddini V, et al. Brief report: fungal balls in the urinary tract of an infant. Indian J Nephrol. 2017;27:165-166. doi:10.4103/ 0971-4065.200517

14. Khaertynov KS, Anokhin VA, Rizvanov AA, et al. Virulence factors and antibiotic resistance of Klebsiella pneumoniae strains isolated from neonates with sepsis. Front Med. 2018;5:225. doi:10.3389/fmed.2018.00225

15. Rodrigues LDS, Rodrigues LDS, Costa LC, et al. Trend in infant mortality rate caused by sepsis in Brazil from 2009 to 2018 . Rev Inst Med Trop Sao Paulo. 2021;63:e26. doi:10.1590/s1678-9946202163026

16. Liu C, Zhang Y, Shang Y, et al. Clinical values of common biomarkers for efficacy monitoring of antibiotics in early-onset neonatal sepsis. Transl Pediatr. 2020;9:669-676. doi:10.21037/tp-20-326

17. Carr D, Barnes EH, Gordon A, et al. Effect of antibiotic use on antimicrobial antibiotic resistance and late-onset neonatal infections over 25 years in an Australian tertiary neonatal unit. Arch Dis Child Fetal Neonatal Ed. 2017;102:F244-F250. doi:10.1136/archdischild-2016-310905

18. Sharma CM, Agrawal RP, Sharan H, et al. "Neonatal sepsis": bacteria \& their susceptibility pattern towards antibiotics in neonatal intensive care unit. J Clin Diagn Res. 2013;7:2511-2513. doi:10.7860/JCDR/2013/6796.3594

19. Moges F, Eshetie S, Yeshitela B, et al. Bacterial etiologic agents causing neonatal sepsis and associated risk factors in Gondar, Northwest Ethiopia. BMC Pediatr. 2017;17:137. doi:10.1186/s12887-017-0892-y

20. Thapa S, Sapkota LB. Changing trend of neonatal septicemia and antibiotic susceptibility pattern of isolates in Nepal. Int J Pediatr. 2019;2019:3784529. doi:10.1155/2019/3784529

21. Muley VA, Ghadage DP, Bhore AV. Bacteriological profile of neonatal septicemia in a tertiary care hospital from Western India. J Glob Infect Dis. 2015;7:75-77. doi:10.4103/0974-777X.154444

22. Li X, Ding X, Shi P, et al. Clinical features and antimicrobial susceptibility profiles of culture-proven neonatal sepsis in a tertiary children's hospital, 2013 to 2017. Medicine. 2019;98:e14686. doi:10.1097/MD.0000000000014686

23. Ballot DE, Nana T, Sriruttan C, et al. Bacterial bloodstream infections in neonates in a developing country. ISRN Pediatr. 2012;2012:508512. doi: $10.5402 / 2012 / 508512$

24. Laxminarayan R, Matsoso P, Pant S, et al. Access to effective antimicrobials: a worldwide challenge. Lancet. 2016;387:168-175. doi:10.1016/ S0140-6736(15)00474-2

25. Russell AB, Sharland M, Heath PT. Improving antibiotic prescribing in neonatal units: time to act. Arch Dis Child Fetal Neonatal Ed. 2012;97: F141-F146. doi:10.1136/adc.2007.120709

26. Xu Xiaohui HB, Baoying T, Huimei L. Clinical study of group B streptococcal septicemia in neonates. J Chin Mod Doctors. 2020;58:90-93. 
27. Daniels JP, Gray J, Pattison HM, et al. Intrapartum tests for group B streptococcus: accuracy and acceptability of screening. Bjog. 2011;118:257-265. doi:10.1111/j.1471-0528.2010.02725.x

28. Chu SM, Hsu JF, Lee CW, et al. Neurological complications after neonatal bacteremia: the clinical characteristics, risk factors, and outcomes. PLoS One. 2014;9:e105294. doi:10.1371/journal.pone.0105294

29. Dong Y, Jiang SY, Zhou Q, et al. Group B Streptococcus causes severe sepsis in term neonates: 8 years experience of a major Chinese neonatal unit. World J Pediatr. 2017;13:314-320. doi:10.1007/s12519-017-0034-5

30. Darlow B, Campbell N, Austin N, et al. The prevention of early-onset neonatal group B streptococcus infection: New Zealand consensus guidelines 2014. NZ Med J. 2015;128:69-76.

31. Schrag SJ, Verani JR. Intrapartum antibiotic prophylaxis for the prevention of perinatal group B streptococcal disease: experience in the United States and implications for a potential group B streptococcal vaccine. Vaccine. 2013;31:D20-D26. doi:10.1016/j.vaccine.2012.11.056

32. Mueller M, Henle A, Droz S, et al. Intrapartum detection of Group B streptococci colonization by rapid PCR-test on labor ward. Eur J Obstet Gynecol Reprod Biol. 2014;176:137-141. doi:10.1016/j.ejogrb.2014.02.039

33. O’Sullivan CP, Lamagni T, Patel D, et al. Group B streptococcal disease in UK and Irish infants younger than 90 days, 2014-15: a prospective surveillance study. Lancet Infect Dis. 2019;19:83-90. doi:10.1016/S1473-3099(18)30555-3

34. Di Renzo GC, Melin P, Berardi A, et al. Intrapartum GBS screening and antibiotic prophylaxis: a European consensus conference. J Matern Fetal Neonatal Med. 2015;28:766-782. doi:10.3109/14767058.2014.934804

35. Nagano N, Nagano Y, Toyama M, et al. Nosocomial spread of multidrug-resistant group B streptococci with reduced penicillin susceptibility belonging to clonal complex 1. J Antimicrob Chemother. 2012;67:849-856. doi:10.1093/jac/dkr546

36. Bauer K, Zemlin M, Hummel M, et al. Diversification of Ig heavy chain genes in human preterm neonates prematurely exposed to environmental antigens. J Immunol. 2002;169:1349-1356. doi:10.4049/jimmunol.169.3.1349

37. Glaser MA, Hughes LM, Jnah A, et al. Neonatal sepsis: a review of pathophysiology and current management strategies. Adv Neonatal Care. 2021;21:49-60. doi:10.1097/ANC.0000000000000769

38. Henneke P, Berner R. SIRS and group-B streptococcal sepsis in newborns: pathogenesis and perspectives in adjunctive therapy. Semin Fetal Neonatal Med. 2006;11:333-342. doi:10.1016/j.siny.2006.03.003

39. Pai S, Enoch DA, Aliyu SH. Bacteremia in children: epidemiology, clinical diagnosis and antibiotic treatment. Expert Rev Anti Infect Ther. 2015;13:1073-1088. doi:10.1586/14787210.2015.1063418

40. Qian L. Clinical characteristics of group B streptococcal septicemia in neonates. Chin Foreign Med Res. 2018;16:136-137.

41. Ashokcoomar P, Bhagwan R. The forgotten needs of mothers during neonatal transfers: a quest for greater sensitivity. $S$ Afr Fam Pract. 2020;62: e1-e8. doi:10.4102/safp.v62i1.5091

42. Arthur KR, Kelz RR, Mills AM, et al. Interhospital transfer: an independent risk factor for mortality in the surgical intensive care unit. Am Surg. 2013;79:909-913. doi:10.1177/000313481307900929

43. Odetola FO, Gebremariam A. Transfer hospitalizations for pediatric severe sepsis or septic shock: resource use and outcomes. BMC Pediatr. 2019;19:196. doi:10.1186/s12887-019-1577-5

44. Sample M, Acharya A, O'Hearn K, et al. The relationship between remoteness and outcomes in critically ill children. Pediatr Crit Care Med. 2017;18:e514-e520. doi:10.1097/PCC.0000000000001318

45. Falsaperla R, Vitaliti G, Amato B, et al. Observational study on the efficiency of Neonatal Emergency Transport in reducing mortality and morbidity indexes in Sicily. Sci Rep. 2021;11:20235. doi:10.1038/s41598-021-99477-5

Infection and Drug Resistance

Dovepress

\section{Publish your work in this journal}

Infection and Drug Resistance is an international, peer-reviewed open-access journal that focuses on the optimal treatment of infection (bacterial, fungal and viral) and the development and institution of preventive strategies to minimize the development and spread of resistance. The journal is specifically concerned with the epidemiology of antibiotic resistance and the mechanisms of resistance development and diffusion in both hospitals and the community. The manuscript management system is completely online and includes a very quick and fair peer-review system, which is all easy to use. Visit http://www.dovepress.com/testimonials.php to read real quotes from published authors.

Submit your manuscript here: https://www.dovepress.com/infection-and-drug-resistance-journal 